\title{
Wind direction over the ocean determined by an airborne, imaging, polarimetric radiometer system
}

\author{
Laursen, Brian; Skou, Niels
}

Published in:

I E E E Transactions on Geoscience and Remote Sensing

Link to article, DOI:

$10.1109 / 36.934086$

Publication date:

2001

Document Version

Publisher's PDF, also known as Version of record

Link back to DTU Orbit

Citation (APA):

Laursen, B., \& Skou, N. (2001). Wind direction over the ocean determined by an airborne, imaging, polarimetric radiometer system. I E E E Transactions on Geoscience and Remote Sensing, 39(7), 1547 - 1555.

https://doi.org/10.1109/36.934086

\section{General rights}

Copyright and moral rights for the publications made accessible in the public portal are retained by the authors and/or other copyright owners and it is a condition of accessing publications that users recognise and abide by the legal requirements associated with these rights.

- Users may download and print one copy of any publication from the public portal for the purpose of private study or research.

- You may not further distribute the material or use it for any profit-making activity or commercial gain

- You may freely distribute the URL identifying the publication in the public portal

If you believe that this document breaches copyright please contact us providing details, and we will remove access to the work immediately and investigate your claim 


\title{
Wind Direction over the Ocean Determined by an Airborne, Imaging, Polarimetric Radiometer System
}

\author{
Brian Laursen, Member, IEEE, and Niels Skou, Senior Member, IEEE
}

\begin{abstract}
The speed and direction of winds over the ocean can be determined by polarimetric radiometers. This has been established by theoretical work and demonstrated experimentally using airborne radiometers carrying out circle flights and thus measuring the full $360^{\circ}$ azimuthal response from the sea surface. An airborne experiment, with the aim of measuring wind direction over the ocean using an imaging polarimetric radiometer, is described. A polarimetric radiometer system of the correlation type, measuring all four Stokes brightness parameters, is used. Imaging is achieved using a 1-m aperture conically scanning antenna. The polarimetric azimuthal signature of the ocean is known from modeling and circle flight experiments. Combining the signature with the measured brightness data from just a single flight track enables the wind direction to be determined on a pixel-by-pixel basis in the radiometer imagery.
\end{abstract}

Index Terms-Microwave radiometry, ocean wind measurements, polarimetric radiometers, remote sensing.

\section{INTRODUCTION}

$\mathbf{O}$ CEAN WIND direction can be assessed with polarimetric radiometer systems measuring the full set of Stokes parameters. Airborne experiments and model work have been carried out, and such activities are still ongoing. Most experimental work has been performed employing a staring radiometer pointing toward the sea surface while the aircraft makes full $360^{\circ}$ turns. Thus, the radiometric signatures as a function of azimuthal observation angle relative to the wind direction were investigated [1]-[4].

Finding the complete $360^{\circ}$ polarimetric signature using a staring radiometer with a long integration time, and hence good radiometric sensitivity, is a necessary and important task, but it is a quite different task to determine the wind direction pixel for pixel based on data from a future spaceborne imager with its inherent short integration time and hence, poorer sensitivity. Furthermore, the full $360^{\circ}$ response will not be available, and the retrieval must rely on experimentally determined or modeled signatures.

Airborne experiments, designed to simulate the space instrument situation, are highly warranted and are the subject of the present paper. A very successful intermediate step was reported in 1998 [5]. Here, the objective was also to retrieve wind directions from airborne imaging radiometer data, but in this case, the

Manuscript received March 9, 2000; revised March 7, 2001. This work was sponsored by in part the Danish National Research Foundation through its support for the Danish Center for Remote Sensing, the Royal Danish Air Force, and Mærsk Olie og Gas, on behalf of Danmarks Undergrunds Consortium (DUC).

The authors are with Oersted DTU, Electromagnetic Systems Section, Technical University of Denmark, DK-2800 Lyngby, Denmark (e-mail: ns@oersted.dtu.dk).

Publisher Item Identifier S 0196-2892(01)05466-3. full $360^{\circ}$ signatures were available due to a unique scan configuration. The experiment to be discussed in the following is rather realistic as the imager only has a limited view of the scene and the wind directions are retrieved pixel for pixel only using the associated azimuthal observation angle.

\section{BACKGROUND}

The present paper dealing with airborne experiments is to be regarded as part 2 of the paper "Polarimetric Radiometer Configurations: Potential Accuracy and Sensitivity" by Skou et al. [6]. In this paper, polarimetry using the Stokes vector notation is discussed as well as basic radiometer configurations and their merits. Also, the airborne radiometer system to be used in the experiments is described. In the following, only a brief summary of that information will be outlined.

The (brightness) Stokes vector is

$$
\begin{aligned}
\bar{T}_{B} & =\left(\begin{array}{c}
I \\
Q \\
U \\
V
\end{array}\right)=\left(\begin{array}{c}
T_{V}+T_{H} \\
T_{V}-T_{H} \\
T_{45^{\circ}}-T_{-45^{\circ}} \\
T_{\ell}-T_{r}
\end{array}\right) \\
= & \frac{\lambda^{2}}{k \cdot z}\left(\begin{array}{c}
\left\langle E_{V}^{2}\right\rangle+\left\langle E_{H}^{2}\right\rangle \\
\left\langle E_{V}^{2}\right\rangle-\left\langle E_{H}^{2}\right\rangle \\
2 \operatorname{Re}\left\langle E_{V} E_{H}^{*}\right\rangle \\
2 \operatorname{Im}\left\langle E_{V} E_{H}^{*}\right\rangle
\end{array}\right)
\end{aligned}
$$

where

$z \quad$ impedance of the medium in which the wave propagates;

$\lambda \quad$ wavelength;

$k \quad$ Boltzmanns constant;

$T_{V} \quad$ vertical;

$T_{H} \quad$ horizontal brightness temperature;

$T_{45^{\circ}}$ and $T_{-45^{\circ}}$ orthogonal linearly polarized measurements skewed $45^{\circ}$ with respect to normal;

$T_{\ell}$ and $T_{r} \quad$ left-hand and right-hand circular polarized quantities;

I total power;

Q difference of the vertical and horizontal power components.

The first and second Stokes parameters are measured using vertically and horizontally polarized radiometer channels, followed by addition or subtraction of the measured brightness temperatures.

The third Stokes parameter can be measured with a two-channel radiometer connected to an orthogonally polarized 
Q

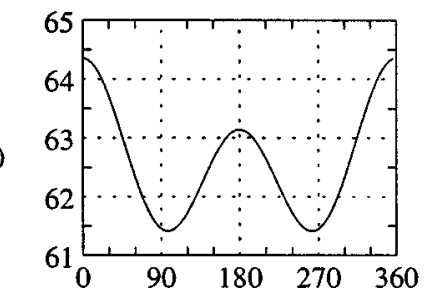

$\mathrm{U}$

$(\mathrm{K})$

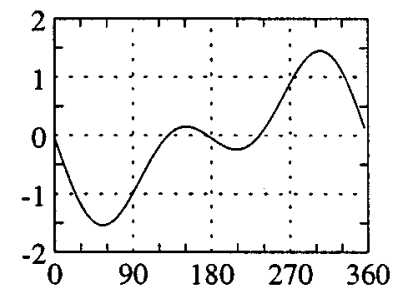

$\mathrm{V}$

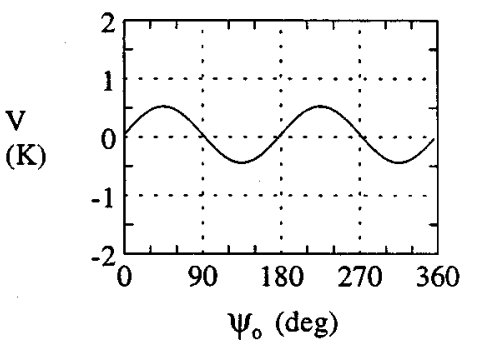

Fig. 1. Typical variation in ocean Stokes parameters with azimuth angle $\psi_{\circ}$. Radiometer frequency is $16 \mathrm{GHz}$ and incidence angle is $55^{\circ}$.

antenna skewed $45^{\circ}$ and subtracting the measured brightness temperatures, or it can be found as the real part of the cross correlation of the vertical and horizontal electrical fields. The fourth Stokes parameter can be measured with the two-channel radiometer connected to a left-hand/right-hand polarized antenna system, or it can be found as the imaginary part of the cross correlation of the vertical and horizontal electrical fields.

All Stokes parameters are simultaneously measured by a twochannel correlation radiometer (employing a complex correlator) connected to a traditional horizontally and vertically polarized antenna system. This is how the radiometer system used here operates.

The measurement of the ocean Stokes parameters place stringent requirements to the sensitivity and accuracy of the radiometers.

Typical variations in the second, third, and fourth Stokes parameters due to wind direction are shown in Fig. 1 . The $x$-axis is the azimuth angle $\psi_{o}$ normalized to the wind direction so that $\psi_{o}=0$ means that the radiometer looks in the upwind direction. The incidence angle is $55^{\circ}$, and the frequency is $16 \mathrm{GHz}$. The curves are based on results from previous experiments [1]-[4]. The second Stokes parameter, $Q$, has a typical variation of $3 \mathrm{~K}$ around a mean value of $63 \mathrm{~K}$. This mean value is dependent on frequency, wind speed, atmospheric attenuation, and other geophysical parameters. The curve has a first and second harmonic cosine shape with maximum in the upwind direction (local maximum downwind) and minimum in crosswind. $U$ has the same typical peak to peak variation but around zero mean. It has a first and second harmonic sine shape with steep zero crossing in the upwind direction and maximum and minimum close to crosswind. The fourth Stokes parameter signal is always somewhat smaller than $Q$ and $U$ but with a very clean second harmonic sine shape.

The airborne, imaging, polarimetric EMIRAD system employs $\mathrm{Ku}(16 \mathrm{GHz})$ and $\mathrm{Ka}(34 \mathrm{GHz})$-band polarimetric radiometers of the correlation type. Each correlation radiometer uses two receivers that are connected to the vertical and the horizontal outputs of a dual-polarized feed horn, analog detectors to find the first two Stokes parameters, and a fast complex digital correlator to find the third and fourth Stokes parameters. The radiometric sensitivity ( $8 \mathrm{~ms}$ integration time) for the vertical (or horizontal) channel is $0.35 \mathrm{~K}$. Thus, the sensitivity for the first and second Stokes parameters is $\sqrt{ } 2 \cdot 0.35=0.5 \mathrm{~K}$. The sensitivity of the correlation measurements, i.e., the third and fourth Stokes parameters, is $0.57 \mathrm{~K}$ ( $8 \mathrm{~m}$ integration).

The imaging antenna is based on an offset $1 \mathrm{~m}$ aperture parabolic reflector scanning sinusoidally around a vertical axis, and illuminated by microwave horns pointing upwards along this axis. The beamwidth of the antenna is $2^{\circ}$ at $\mathrm{Ku}$ band and $1^{\circ}$ at Ka band. The cross polarization due to the offset parabolic reflector is below $25 \mathrm{~dB}$. The maximum scan angle is $\pm 25^{\circ}$, and the incidence angle on the ground is constantly $55^{\circ}$ at $\mathrm{Ku}$ band and $50^{\circ}$ at Ka band for this conically scanned system. Due to the fixed feed horn and the scanning reflector, significant polarization mixing takes place when scanning away from straight aft. This is corrected in the data analysis, following the considerations in [7].

The antenna and the receivers are mounted on a cargo pallet, which is positioned on the loading ramp of a $\mathrm{C}-130$ aircraft. The ramp is closed during takeoff, landing, and transit, but lowered to its horizontal position when measurements are to be carried out. The antenna thus has an unobstructed view of the scene below and aft of the aircraft. The ground speed of the aircraft is $70 \mathrm{~m} / \mathrm{s}$ during operation with the ramp open. The flight altitude is $2000 \mathrm{~m}$, and the $\pm 25^{\circ}$ scan then results in a swath of 2000 $\mathrm{m}$. For the $2000 \mathrm{~m}$ altitude the footprint on ground is $94 \times 146$ $\mathrm{m}$ at Ku band, and $48 \times 74 \mathrm{~m}$ at Ka band. $8 \mathrm{~ms}$ sampling and integration time in the radiometers in combination with a $2 \mathrm{~s}$ scan period ensure contiguous imaging at the highest frequency.

The polarimetric signatures of the wind-driven sea only show small azimuthal variations in the range of a few $\mathrm{K}$. At the same time, they are quite dependent on incidence angle and pointing geometry, which may result in polarization rotation. Thus, aircraft attitude must be carefully monitored, and the radiometer system includes an inertial navigation unit mounted directly on the antenna frame. The antenna attitude is thus measured to within $1 / 10^{\circ}$. Using this information, data is corrected for unwanted attitude variations (typically less than $1^{\circ}$ in the present case).

\section{OCEAN WIND RETRIEVAL}

A polarimetric radiometer system for ocean wind vector measurements will typically use several frequencies (here $\mathrm{Ku}$ and Ka band), and the three Stokes parameters $Q, U$, and $V$. Some signals will be strongly dependent, for example $U$, at $\mathrm{Ku}$ and $\mathrm{Ka}$ band, while others will be orthogonal like $Q$ and $U$ at the same frequency. The wind direction retrieval is theoretically completely free of direction ambiguities due to this orthogonality. 
But noise, be it radiometric or from other sources, will give uncertainties in the retrieval, especially in the downwind directions where the $Q$ and $U$ signal variations are smallest. The resulting errors in wind direction do not just resemble Gaussian noise but include possible ambiguities, i.e., directions way off like for example $140^{\circ}$. There will be more about this in Section VII about error analysis.

In order to carry out the wind retrieval from a given set of polarimetric radiometer measurements, the azimuthal signature of the sea has to be established corresponding to the actual conditions of the measurements: frequency, incidence angle, atmospheric conditions, sea temperature, wind speed. This is called the model function, and it will typically look like Fig. 1. It can be determined by modeling or by carrying out a proper $360^{\circ}$ circle flight on the day of the measurement. The first is what is needed in a future operational system, while the latter is a viable option in the experimental phase. The wind retrieval used here is based on a nonlinear weighted least-squares minimization of the following error expression:

$$
e\left(\psi_{0}\right)=\sum_{j=1}^{n} \sum_{i=1}^{4} \frac{\left(\bar{T}_{i, j}\left(\psi_{0}\right)-T_{i, j}\right)^{2}}{\Delta T_{i, j}^{2}}
$$

where

$$
\begin{array}{ll}
n & \text { number of frequency bands used (here 2), } \\
\bar{T}_{i, j}\left(\psi_{0}\right) & \text { model function and } \\
T_{i, j} & \text { measurement at the } j \text { th frequency concerning the } \\
& \begin{array}{l}
\text { ith Stokes parameter (here only the second, third, } \\
\text { and forth). }
\end{array}
\end{array}
$$

The weighting functions $\Delta T_{i, j}$ does not only include radiometric noise, but also noise from uncertainties in antenna pointing (incidence angle, polarization mixing). Thus, in the retrieval, preference is given to less noisy channels.

It was found of advantage to make a slight modification to the error expression in order to reflect the fact that some Stokes parameters have larger peak to peak response $\left(\bar{T}_{i, j p p}\right)$ than others, that is, put extra weight to channels with large response. Hence, in the present case the error function is

$$
e\left(\psi_{0}\right)=\sum_{j=1}^{2} \sum_{i=2}^{4} \frac{\left(\bar{T}_{i, j}\left(\psi_{0}\right)-T_{i, j}\right)^{2}}{\Delta T_{i, j}^{2}} \cdot\left(\bar{T}_{i, j p p}\right)^{2} .
$$

$I$ is very dependent on cloud conditions (atmospheric attenuation), $Q$ is quite dependent on cloud conditions, while $U$ and $V$ are only marginally dependent on clouds. This is not surprising bearing in mind that $I$ is the sum of $T_{V}$ and $T_{H}$, both being dependent on atmospheric conditions. $Q$ is the difference between $T_{V}$ and $T_{H}$, but changes due to varying atmospheric conditions do not quite cancel since $T_{V}$ is much larger than $T_{H}$ (typically $63 \mathrm{~K}$, see Fig. 1). In contrast to this, $U$ and $V$ can be interpreted as differences between channels having almost the same signal strength (see the Stokes vector definition and Fig. 1: $U$ and $V$ has a zero mean value). Thus, for example, $T_{45^{\circ}}$ increases with atmospheric attenuation, but $T_{-45^{\circ}}$ will increase with the same amount, and $U$ will to first order be unaffected. Therefore, in cloudy conditions with unusually severe attenuation (actually monitored using the $I$ channel), the weighting function for $Q\left(\Delta T_{2, j}\right)$ was enhanced proportionally to put less weight on $Q$ in the retrieval (rely more on $U$ and $V$ ). In practice, this was done by comparing the measured $I$ values with the expected $I$ values, calculated using the actual wind speed, sea temperature, salinity, but standard atmosphere without clouds. For differences $0-20 \mathrm{~K}, \Delta T_{2, j}$ was enhanced proportionally resulting eventually in a weight on $Q$ as low as 5\% compared with $U$ and $V$.

In short, the retrieval procedure is the following.

- Establish the model function $\bar{T}_{i, j}\left(\psi_{0}\right)$.

- Establish the peak to peak response $\bar{T}_{i, j p p}$.

- Establish the weighting function $\Delta T_{i, j}$.

- Compare the measurements $T_{i, j}$ with the model function (properly weighted) and find the wind angle $\psi_{0}$ that results in the smallest difference $e\left(\psi_{0}\right)$.

\section{WeATHER CONDITIONS AND Flight PATtern}

The polarimetric radiometer data to be discussed in the following sections were acquired on October 21 and November 27,1998 over a target area centered at $55^{\circ} 40^{\prime} \mathrm{N}, 4^{\circ} 46^{\prime} \mathrm{E}$ in the middle of the North Sea. The test site was chosen between two offshore oil platforms from where in situ wind data was acquired. One platform was approximately $8 \mathrm{~km}$ to the north of the target center, the other $8 \mathrm{~km}$ to the south. Thus wind shadow effects in the test area, stemming from the oil platforms, are avoided under predominantly westerly winds.

On October 21, at the time of data collection, the winds in the area were $17 \mathrm{~m} / \mathrm{s}$ with a direction of $216^{\circ}$. The wind speed and direction were quite stable for many hours before and during the experiment. The winds are normalized to standard meteorological reference, and they are $5 \mathrm{~min}$ averages disregarding gusts. Cloud conditions were overcast with heavy clouds and even showers.

On November 27, the wind conditions were less favorable. At the time of data collection (around noon local time) the wind speed was $6 \mathrm{~m} / \mathrm{s}$ with a $110^{\circ}$ direction, but during the morning, the wind had dropped gradually from $10 \mathrm{~m} / \mathrm{s}, 330^{\circ}$ to a zero wind speed and direction jump to $110^{\circ}$ just one hour prior to the experiment. This behavior is associated with the passage of a frontal system also responsible for poor cloud conditions: overcast with heavy clouds and showers.

Fig. 2 shows details about the flight pattern. The left-hand part of the figure summarize the imaging parameters, while the right-hand part shows the actual flight pattern centered at the target center coordinates. Eight passes are carried out, four legs each flown back and forth. Each pass is approximately 8 $\mathrm{km}$. The eight passes correspond to headings: 1) $270^{\circ}$, 2) $90^{\circ}$, 3) $315^{\circ}$, 4) $135^{\circ}$, 5) $\left.00^{\circ}, 6\right) 180^{\circ}$, 7) $45^{\circ}$, and 8) $225^{\circ}$. Flying the passes two by two back and forth simulates a possible fore-and-aft look situation for a future spaceborne instrument. The time delay between two observations of the target center is around $5 \mathrm{~min}$, fitting well with the time between the fore and the aft look for a typical space instrument. During the passes, aircraft roll and pitch are kept to a minimum, generally below a few tenths of a degree.

In addition to the primary flight pattern described above, a circle flight was carried out to check the instruments and to confirm the model function. Such measurements are carried out with the radiometers staring at the sea surface without scanning, while the aircraft makes full $360^{\circ}$ turns around the target center. Actually, in the present case, these measurements are used to establish the mean value of the second Stokes parameter 

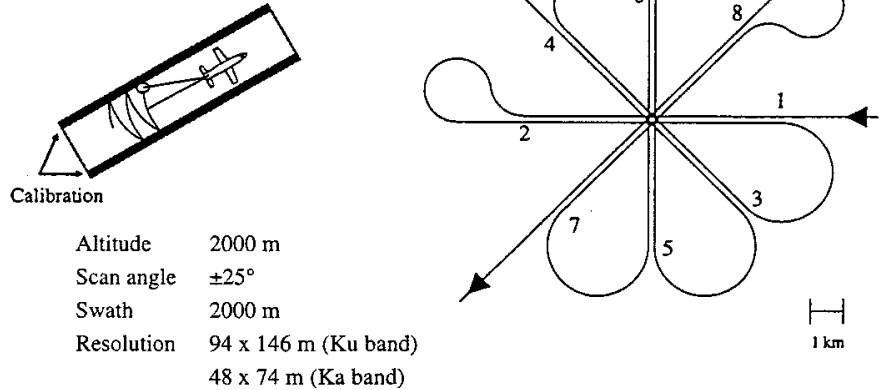

Fig. 2. Imaging geometry and flight pattern.

(K)

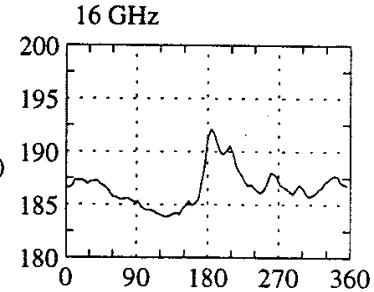

Th

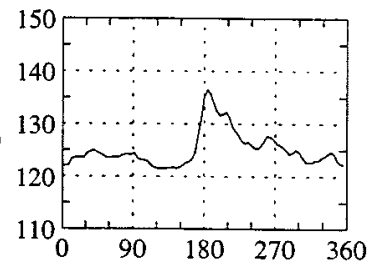

Q

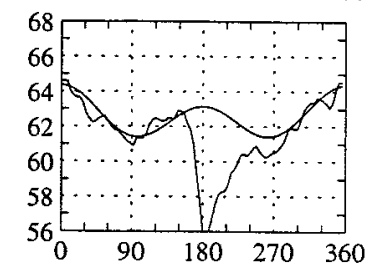

$\mathrm{U}$

(K)
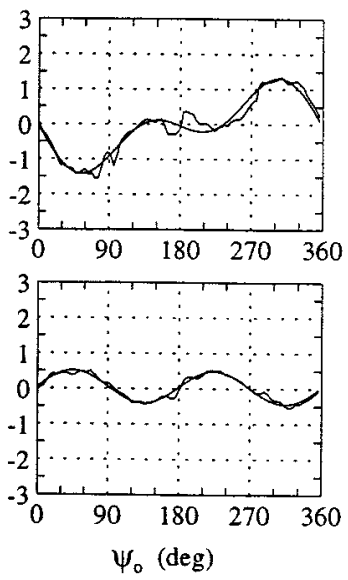

Fig. 3. The $16 \mathrm{GHz}$ circle flight results: $10 \mathrm{~s}$ integration time.

model function as this is dependent on atmospheric conditions not being measured otherwise.

\section{Circle Flight}

As an example, $16 \mathrm{GHz}$ circle flight data from October 21 is shown in Fig. 3. The brightness temperatures and the Stokes
(K)
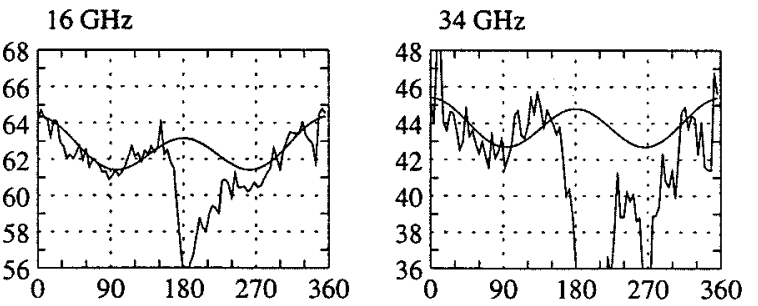

$\mathrm{U}$
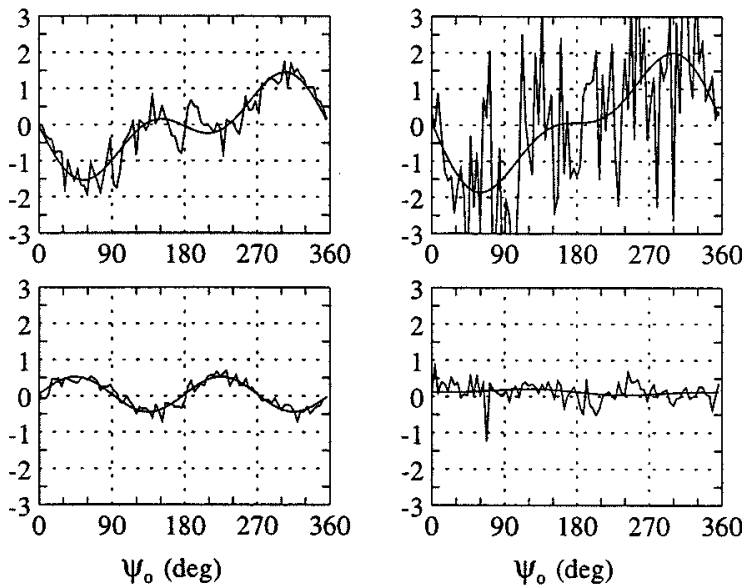

Fig. 4. The 16 and $34 \mathrm{GHz}$ circle flight results: $1 \mathrm{~s}$ integration time.

parameters are shown as functions of the relative wind direction $\psi_{o}$, which is the angle between the wind direction and the radiometer observation azimuth angle so that $\psi_{0}=0$ is upwind. Data is averaged for $10 \mathrm{~s}$, during which the aircraft moves $700 \mathrm{~m}$ along track, and the original $94 \times 146 \mathrm{~m}$ footprint is elongated to a $94 \times 846 \mathrm{~m}$ resolution cell. The smooth first and second harmonic cosine and sine curves are based on modeling as previously described, and the $Q$ curve has been offset to fit the data (thus accounting for the general atmospheric attenuation of the day).

The influence of atmospheric conditions as discussed in Section III is quite obvious. At the time corresponding to azimuth angles around $180^{\circ}$, a heavy cloud is encountered. The vertical and horizontal brightness temperatures, and hence the first (not shown here) and second Stokes parameters, are strongly corrupted by this cloud, while the third and fourth Stokes parameters are only marginally influenced. It is evident that use of the second Stokes parameter for wind retrieval under such conditions would lead to faulty results. This is partly avoided by the weighting as described in Section III.

It is of interest to observe the influence of a relatively small footprint being of a size comparable with features on the windy sea surface. Fig. 4 shows partly the same data as presented in Fig. 3, only this time only integrated $1 \mathrm{~s}$. The Stokes parameters $Q, U$, and $V$ are shown, and this time both at 16 and 34 $\mathrm{GHz}$. The footprints are approximately $94 \times 216 \mathrm{~m}$ at $16 \mathrm{GHz}$ and $48 \times 144 \mathrm{~m}$ at $34 \mathrm{GHz}$. A noisy appearance is evident, especially concerning the $U$ parameter at $34 \mathrm{GHz}$. Radiometric noise is with $1 \mathrm{~s}$ integration time around $0.05 \mathrm{~K}$ and thus insignificant. Residual uncorrected attitude errors would influence both frequencies approximately equally. It has been observed that increasing atmospheric attenuation dampen the noise-like signal, especially at $34 \mathrm{GHz}$. It is thus concluded that the signal is a true sea-surface signal ("geophysical noise") being significantly 

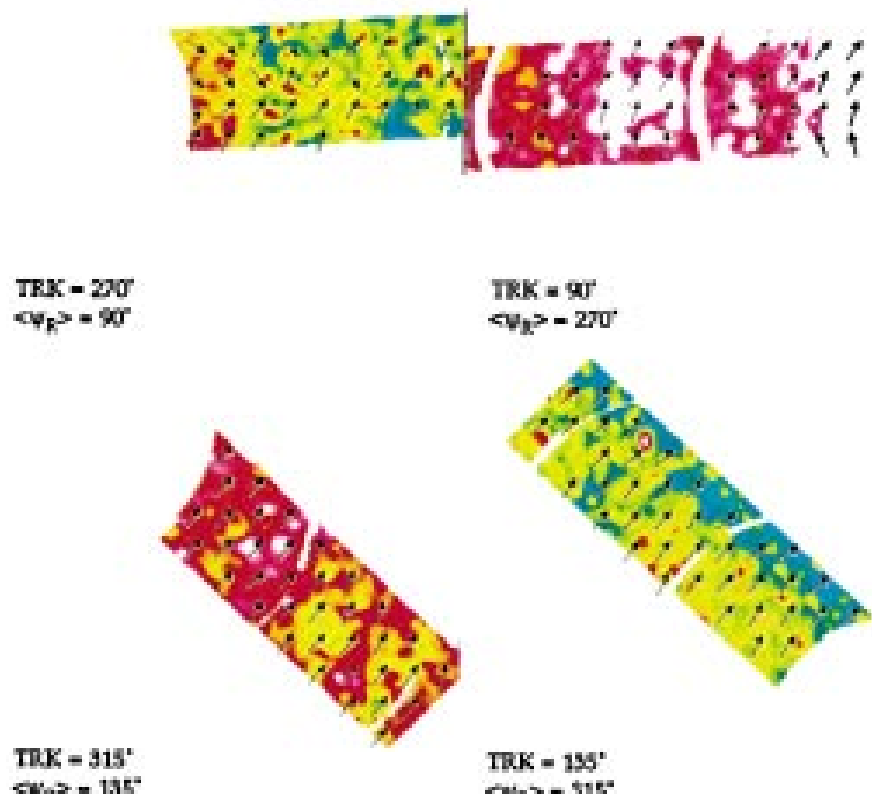

$\langle\psi\rangle=135^{\circ}$
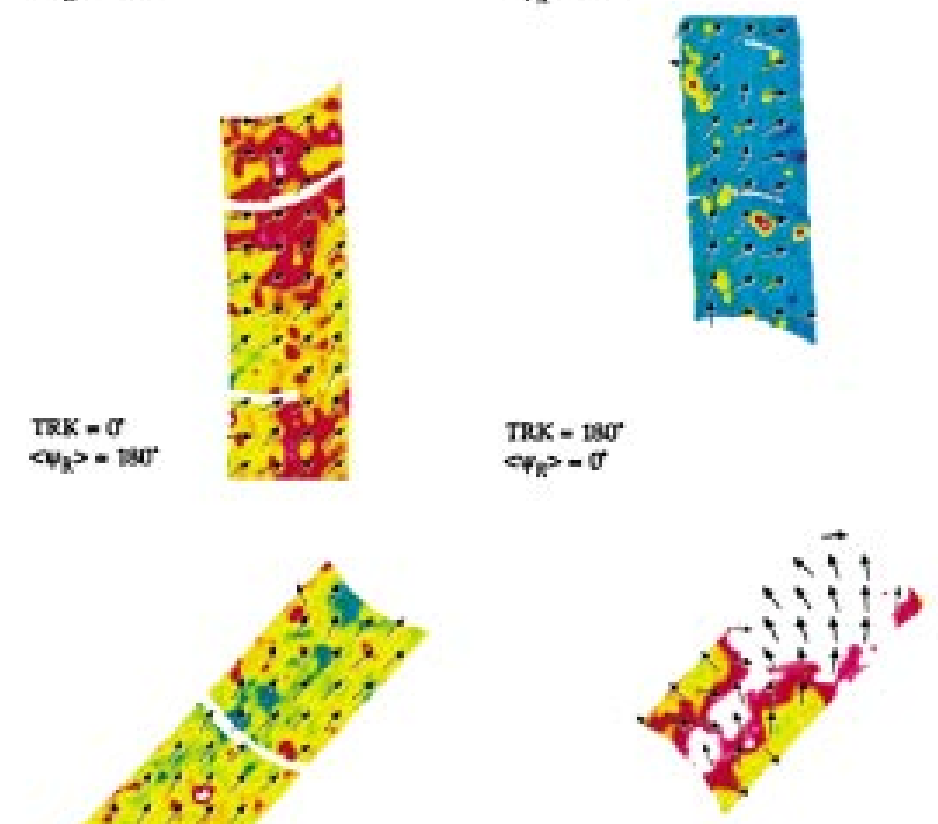

TRK $=225$

$\left\langle\psi_{\mathrm{a}}\right\rangle=\mathbf{4}^{\prime}$
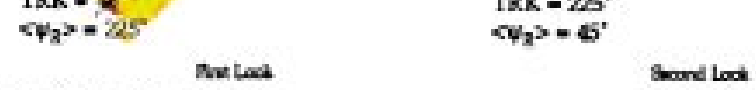

TRK $=9 \mathbf{S O}^{5}$

$4 x_{x}>-2 \pi 0$

TRK $=195^{\circ}$
$\left\langle\psi_{a}\right\rangle=-515^{\circ}$

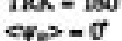
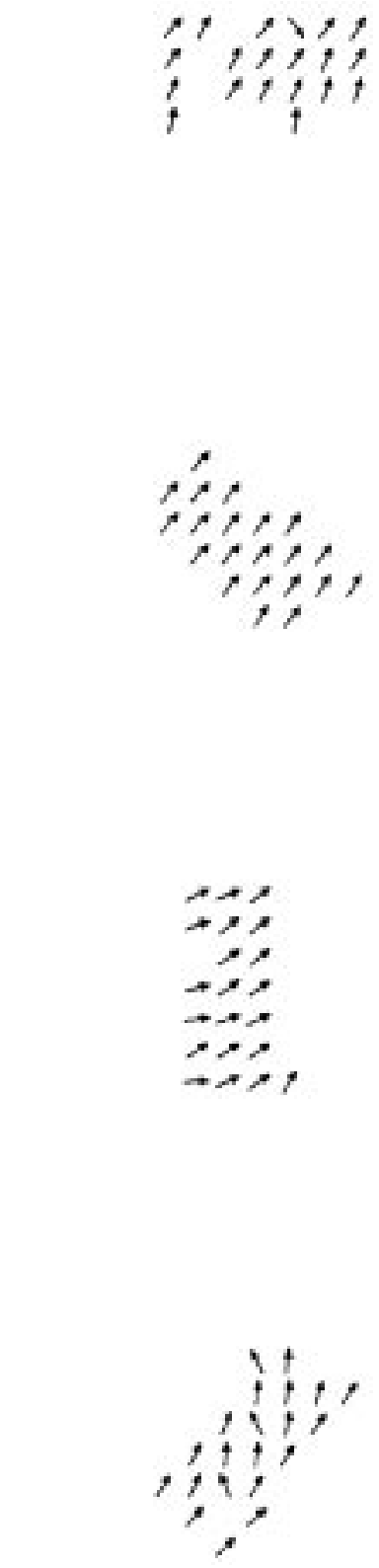

Noth Sea, Denmark

Acquired: 1996.1021 - Station 1
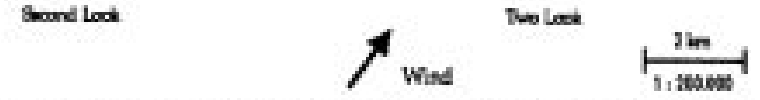

DCRS, Dept. of Electromagnetic Systems, Technical University of Denmark

Fig. 5. Radiometric $I$ data and retrieved winds from the October 21 flight. TRK is aircraft track angle, while $\left\langle\psi_{R}\right\rangle$ is average azimuth look angle (TRK-180 ${ }^{\circ}$ due to aft-looking).

stronger at $34 \mathrm{GHz}$ than at $16 \mathrm{GHz}$. We explain it as a perturbation of the Stokes parameter signal due to the fact that the footprint, and especially at $34 \mathrm{GHz}$, is comparable in size to the distance between wave crests. This is supported by the fact that by careful inspection of full resolution (i.e., no postprocessing integration) $U$ imagery at $34 \mathrm{GHz}$, a striped appearance is seen, indicating that the individual wave fronts are being imaged. Any satellite sensor would of course not see this effect, and our data have to be spatially integrated to be realistic and to correctly measure the required Stokes parameters which reflect bulk properties, not properties of individual waves.

\section{IMAGING Mode}

Fig. 5 shows an example of the radiometric data acquired and the retrieved wind directions. The eight panels in the left-hand 
and middle columns correspond to the eight passes over the target area. Recalling that the flight consisted of four legs each flown back and forth, it is seen that the left-hand column can be regarded as four examples of a fore look and the middle column is the corresponding aft looks $\left(180^{\circ}\right.$ apart from the first). The $16 \mathrm{GHz} I$ data is shown as the background, with bluish colors indicating the sea signature, and green-yellow-red-white indicating increasing brightness temperature due to increasing atmospheric attenuation. All panels have north pointing toward the top of the page, and with the target center at the panel center. The recorded swaths are shown properly oriented and located in the appropriate panels. The average in situ wind direction is shown at the bottom of the figure. The wind directions, as retrieved from the recorded radiometric data, are shown as arrows on top of the $16 \mathrm{GHz}$ I data. Before the retrieval, the data was spatially averaged to $600 \times 600 \mathrm{~m}$ ground resolution in order to avoid measurements on individual waves, i.e., obtain average surface conditions. At the same time, radiometric sensitivity is improved with a factor roughly equal to the square root of the ratio between the areas of the averaged resolution cell and the original $\mathrm{Ka}$ band footprint (just corresponding to contiguous ground sampling). In the present case this corresponds to a roughly ten-fold improvement, i.e., to $0.05 \mathrm{~K}$ for $I$ and $Q$, and to $0.057 \mathrm{~K}$ for $U$ and $V$.

The third (right-hand) column is an attempt to combine the wind retrievals from the simulated fore and aft looks into a two-look situation. A rather simple, automatic selection algorithm has been implemented. If the difference between directions of the two single look retrievals is smaller than $60^{\circ}$, the average value has been calculated and plotted (the value $60^{\circ}$ is a rather arbitrary choice, found to work well during the processing of the actual data). Differences larger than $60^{\circ}$ indicate ambiguity problems, and a selection of one direction instead of the other must be performed. As already stated, the upwind condition is favorable compared with the downwind condition due to larger polarimetric signatures in that direction. Hence, if one look has an estimated wind vector in the upwind sector, and the other has one in the downwind sector, the upwind is considered more reliable and hence selected. Note that all these operations are incorporated in the data processing software and are performed automatically, i.e., without human intervention.

In general, the retrieved wind directions compare well with the actual wind direction with relatively few ambiguities. The worst errors and ambiguities are seen in the lower row (45/225 passes), where the second look is seriously hampered by a massive, rainy cloud that shows up as red and white in the underlying I image. An interesting observation can be made in the top row (270/90 passes). The first look has many approximately $90^{\circ} \mathrm{am}-$ biguities due to predominantly downwind look geometry, while the second look has no ambiguities, despite a more severe cloud condition, due to upwind look geometry.

Table I summarizes the wind retrievals.

The combined wind directions, as illustrated in the right-hand column of Fig. 5, have been averaged and the standard deviation calculated. The wind direction is found quite well, but the standard deviation is very sensitive to ambiguities. Looking at the wind vectors in Fig. 5, it is quite obvious that even a very simple "geophysical filter" can remove the ambiguities in most
TABLE I

Wind RETRIEVAL Statistics: TRUe Wind $216^{\circ}$

\begin{tabular}{l|c|c|c|c}
\hline & \multicolumn{2}{|c|}{ all wind vectors } & \multicolumn{2}{c}{ ambiguity removed } \\
\hline passes & wind dir. & stdev. & wind dir. & stdev. \\
\hline $270 / 90$ & $210^{\circ}$ & $26^{\circ}$ & $207^{\circ}$ & $8^{\circ}$ \\
\hline $315 / 135$ & $218^{\circ}$ & $4^{\circ}$ & $218^{\circ}$ & $4^{\circ}$ \\
\hline $0 / 180$ & $240^{\circ}$ & $12^{\circ}$ & $240^{\circ}$ & $12^{\circ}$ \\
\hline $45 / 225$ & $198^{\circ}$ & $22^{\circ}$ & $204^{\circ}$ & $14^{\circ}$ \\
\hline
\end{tabular}

TABLE II

Wind Retrieval Statistics, SECOND EXPERIMENT: TRUe Wind 225

\begin{tabular}{l|c|c|c|c}
\hline & \multicolumn{2}{|c|}{ all wind vectors } & \multicolumn{2}{c}{ ambiguity removed } \\
\hline passes & wind dir. & stdev. & wind dir. & stdev. \\
\hline $270 / 90$ & $221^{\circ}$ & 9 & $221^{\circ}$ & 9 \\
\hline $315 / 135$ & $227^{\circ}$ & $6^{\circ}$ & $227^{\circ}$ & $6^{\circ}$ \\
\hline $0 / 180$ & $230^{\circ}$ & $13^{\circ}$ & $230^{\circ}$ & $13^{\circ}$ \\
\hline $45 / 225$ & $225^{\circ}$ & $63^{\circ}$ & $228^{\circ}$ & $10^{\circ}$ \\
\hline
\end{tabular}

cases: it is not meaningful with one wind vector pointing $90^{\circ}$ away from the others amidst a wind field. Manually removing such obvious ambiguities in the upper and the lower row significantly improves the statistics as seen in the second half of Table I.

The data set presented and discussed above was acquired approximately one hour before noon. Another experiment was carried out $2 \mathrm{~h}$ later on the same day with practically the same weather conditions. Table II summarizes the wind retrievals.

The results are seen to be almost identical to those from the first experiment. On November 27, an experiment was performed under very different wind conditions as mentioned in Section IV. Fig. 6 displays the data in the same format as used in Fig. 5. Significant confusion in the retrieved wind directions is observed in the two upper rows (passes 270/90 and $315 / 135$ ), while a fairly good retrieval is seen in the two lower rows (passes $0 / 180$ and 45/225). In this case, it has no meaning to attempt a simple ambiguity removal concerning the $270 / 90$ and $315 / 135$ passes, while this is absolutely justified and works well for the $0 / 180$ and $45 / 225$ passes. The wind direction is found quite well and with reasonable standard deviation, see Table III summarizing the wind retrievals from the November 27 experiment.

This is quite a pleasing result bearing in mind the difficult situation combining low wind speed, sudden discontinuity in wind direction shortly prior to sensing, and severe cloud condition. It is noteworthy that this observation supports the theory that the polarimetric radiometer senses the actual wind direction disregarding past history.

\section{ERRORS IN THE DIRECTION RETRIEVALS}

Returning to Fig. 5, several erroneous wind directions were noted, and a further discussion of the ambiguity situation will be carried out in the following in a simplified case. Assume 

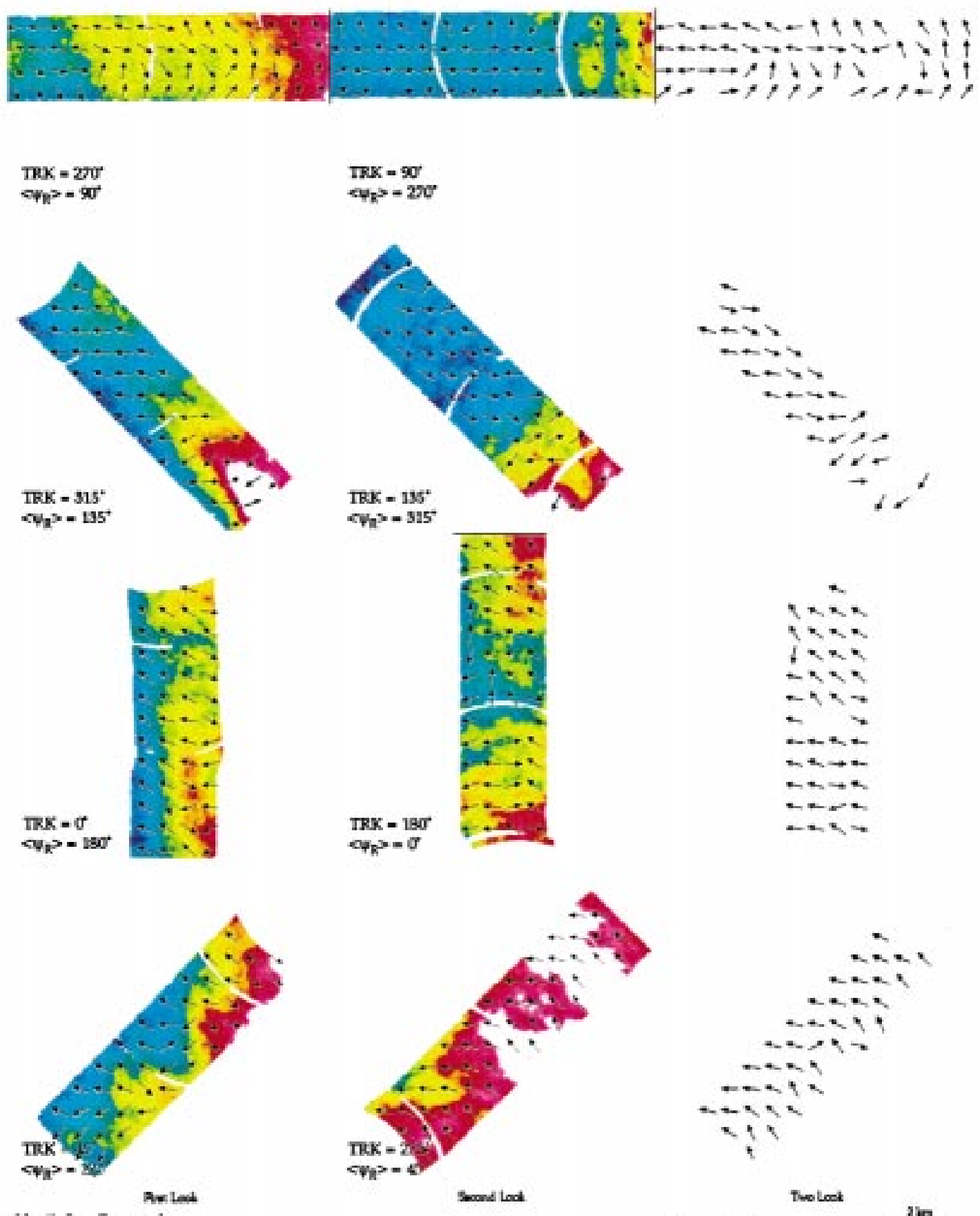

North Sen, Denmark Acquired: 1998.11.27
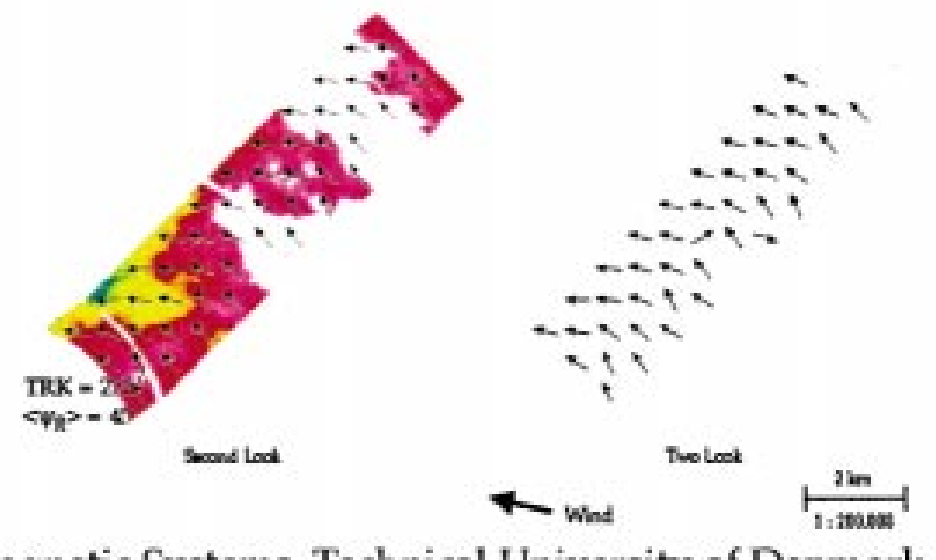

DCRS, Dept. of Electromagnetic Systems, Technical University of Denmark

Fig. 6. Radiometric $I$ data and retrieved winds from the November 27 flight. TRK is aircraft track angle, while $\left\langle\psi_{R}\right)$ is average azimuth look angle (TRK-180 ${ }^{\circ}$ due to aft-looking).

only one frequency, $16 \mathrm{GHz}$, and only two Stokes parameters, namely $\mathrm{Q}$ and $\mathrm{U}$. The model function shown in Fig. 1 is assumed (only $Q$ and $U$ ), and the error function [equation (2) from Section III] reduces to

$$
e\left(\psi_{o}\right)=\left(Q\left(\psi_{o}\right)-Q_{m}\right)^{2}+\left(U\left(\psi_{o}\right)-U_{m}\right)^{2}
$$

assuming equal weight on both channels. $Q\left(\psi_{0}\right)$ and $U\left(\psi_{0}\right)$ are the model functions, while $Q_{m}$ and $U_{m}$ are measured values in a given case.
Let us consider a situation where the values $Q_{m}=Q\left(60^{\circ}\right)$ and $U_{m}=U\left(60^{\circ}\right)$ are measured. For this situation, the error function is illustrated in Fig. 7. The error is 0 at $60^{\circ}$ as expected, and the direction $60^{\circ}$ is found correctly by the procedure outlined in Section III [minimize $\left.e\left(\psi_{o}\right)\right]$. Even rather large offsets or variations in the measured $Q_{m}$ and $U_{m}$ values due to noise or other measurement errors will not cause the second minimum to be the global minimum resulting in a faulty retrieved direction. This is typical for more or less upwind looking cases. 
TABLE III

Wind RETRIEVAl STATISTICS, NOVEMBER EXPERIMENT: TRUE Wind $110^{\circ}$

\begin{tabular}{l|c|c|c|c}
\hline & \multicolumn{2}{|c|}{ all wind vectors } & \multicolumn{2}{c}{ ambiguity removed } \\
\hline passes & wind dir. & stdev. & wind dir. & stdev \\
\hline $270 / 90$ & $188^{\circ}$ & $81^{\circ}$ & & \\
\hline $315 / 135$ & $51^{\circ}$ & $91^{\circ}$ & & \\
\hline $0 / 180$ & $112^{\circ}$ & $62^{\circ}$ & $113^{\circ}$ & $16^{\circ}$ \\
\hline $45 / 225$ & $122^{\circ}$ & $36^{\circ}$ & $120^{\circ}$ & $18^{\circ}$ \\
\hline
\end{tabular}

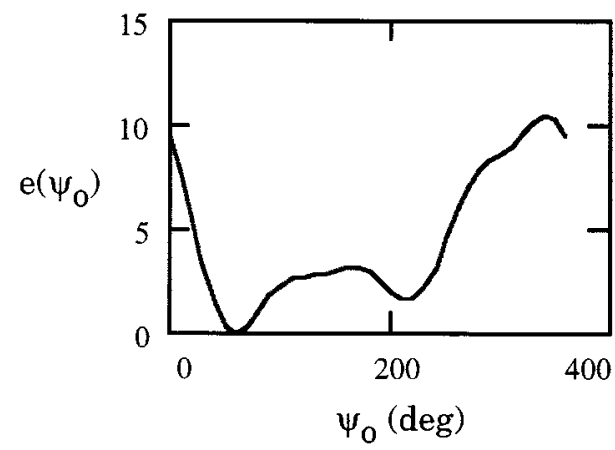

Fig. 7. Retrieval error function $e\left(\psi_{o}\right)$ : true wind at $60^{\circ}$.

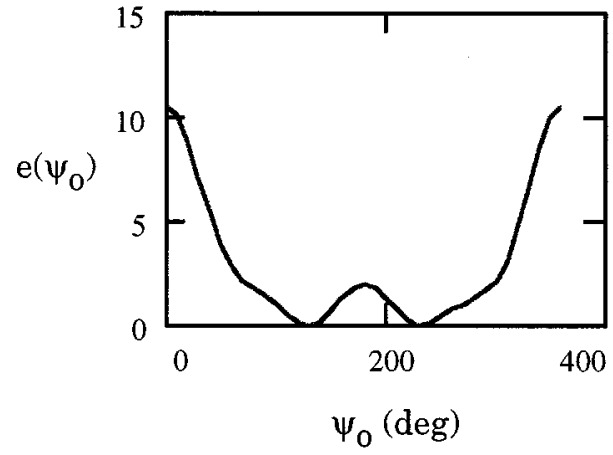

Fig. 8. Retrieval error function $e\left(\psi_{o}\right)$ : true wind at $130^{\circ}$.

If, however, we study a case where we have measured $Q_{m}=$ $Q\left(130^{\circ}\right)$ and $U_{m}=U\left(130^{\circ}\right)$, the situation is quite different, as shown in Fig. 8. The error is 0 at $130^{\circ}$, and this is the global minimum as we would expect, but it is seen that a second very deep minimum is present at $230^{\circ}$. Even a small amount of measurement error can cause the first null to be slightly filled and the second minimum to be enhanced and become the global minimum, and an ambiguity error arises.

Fig. 3 indicates typical measurement errors of $\pm 0.5 \mathrm{~K}$ disregarding obvious clouds, and this corresponds to a $94 \times 846 \mathrm{~m}$ footprint. Fig. 5 represents data from a $600 \times 600 \mathrm{~m}$ footprint, i.e., five times larger, and typical errors are thus estimated to $\pm 0.5 / \sqrt{ } 5= \pm 0.2 \mathrm{~K}$. Returning to the case above, just subtracting $0.2 \mathrm{~K}$ from the $U_{m}$ value causes the $230^{\circ}$ minimum to be very small $(0.000013)$ and the $130^{\circ}$ minimum to be filled to the value 0.04. A wrong (ambiguous) direction is thus found. Notice that the angle values $130^{\circ} / 230^{\circ}$ are symmetrical around the $180^{\circ}$ direction.

Investigating this further reveals that for angles $\psi_{0}$ up to about $100-110^{\circ}$, a relatively robust retrieval is expected where even rather large measurement errors will not cause ambiguities. Above that angular range (i.e., the downwind range of angles), typical errors in the order of $\pm 0.2 \mathrm{~K}$ can cause ambiguities. The ambiguities are symmetrical around the $180^{\circ}$ direction, and for example, a $60^{\circ}$ ambiguity corresponds to the $150^{\circ} / 210^{\circ}$ case

With this in mind, let us return to Fig. 5, upper-left panel (the $270^{\circ}$ pass). The wind direction is $\psi_{w}=216^{\circ}$. Hence, $\psi_{o}=$ $\psi_{w}-\left\langle\psi_{R}\right\rangle=126^{\circ}$. This gives a potential ambiguity $108^{\circ}$ (i.e., slightly more than $90^{\circ}$ ) away from the true wind, which is exactly what is observed in nine cases.

Also in the lower row, pass $225^{\circ}$, significant direction errors are noticed. In this case, a heavy (possibly raining) cloud disturbs the measurement and the weighting on $Q$ in the retrieval is very low. Thus, the previous discussion cannot explain the direction errors. The directions are found primarily using the $U$ and $V$ parameters, and it is noted from the model function in Fig. 1 that in this typical downwind case the variations in $U$ are very small, paving the way for direction errors.

The rather confusing situation in the upper two rows of Fig. 6 has not been analyzed in any detail. A certain randomness is evident and the case is probably close to the threshold concerning SNR. Note that the error analysis as described earlier cannot be applied directly as the model function of Fig. 1 cannot be used for this low wind case.

\section{DISCUSSION AND CONCLUSIONS}

An imaging, polarimetric radiometer system, measuring the full set of Stokes parameters at Ku and Ka band, has been used in airborne measurements over the wind-driven sea. An experiment was carried out over the North Sea on a day with good winds $(17 \mathrm{~m} / \mathrm{s}$ ) but rather poor (but realistic) weather conditions for radiometer measurements: heavy clouds and rain showers. Despite the weather, wind direction was found with good fidelity and few ambiguities in most cases, especially using a combination of two looks $180^{\circ}$ apart, representing a possible space system configuration with a fore and an aft look. Removal of obvious ambiguities, further improves the results, and the wind direction is on average found to be within $8^{\circ}$ of the true value, with a standard deviation of $10^{\circ}$.

Another experiment was carried out under very different wind conditions: only $6 \mathrm{~m} / \mathrm{s}$, but the wind had dropped to zero and jumped $140^{\circ}$ in direction just one hour prior to the experiment. Also, here the weather conditions were difficult with heavy clouds and showers. Despite this, the wind direction was found quite well from some flight tracks to within $9^{\circ}$ of the true value, with a standard deviation of $17^{\circ}$. On other flight tracks, retrieval was not possible, and the situation with poor weather and low winds can be concluded as being marginal for proper wind direction retrieval. An interesting observation is that in the cases where retrieval was possible, the correct wind direction was found despite the wind jump one hour before sensing, confirming that the polarimetric radiometer measures the actual winds and not past history.

From the data, it is also clear that one-look retrieval is possible, with more ambiguities to handle, however. This is of importance when designing a future spaceborne polarimetric 
system: the inclusion of both a fore and an aft look is no problem for the imaging radiometer itself, but it certainly imposes constraints on the spacecraft design to ensure unobscured looks fore and aft. Further analysis is needed in order to assess more accurately the benefits of having two looks for the retrieval. Also note that further work on how to combine the data from the two potential looks is warranted. In the present case, the wind directions have been found independently in the two looks and afterwards averaged to find the two-look solution. This is probably not the optimal way for retrieval. One could combine the data from both looks, enter twice the amount of data into the error function (add an extra summation in equation (3) summing the fore and the aft situation), and thus directly find the two-look wind vector solution that minimizes the error. This way, the wind direction accuracy could well improve and the ambiguity problem be more manageable.

A very simple method was used to remove obvious ambiguities by noting that it is not geophysically meaningful to have a few wind vectors pointing far off the general direction of a host of wind vectors. In the section with the discussion on ambiguity errors, it is seen that the wind direction retrieval algorithm, in addition to giving a certain direction (where the error function is minimum), can also provide potential ambiguous directions as output. This can be used to help solve ambiguities in future applications where wind data from a spaceborne polarimetric radiometer will be merged with numerical weather models. If a suspicious retrieved direction does not fit the model but the possible ambiguous direction does, this can be selected with great confidence, as it is in fact also a possible output of the polarimetric retrieval algorithm.

In this paper, measurements were taken at $\mathrm{Ku}$ and $\mathrm{Ka}$ bands. It has, however, not been thoroughly investigated to which degree the retrieval process benefits from having two frequencies. Bearing in mind that the Stokes parameter model functions are not very different at $\mathrm{Ku}$ and $\mathrm{Ka}$ bands, one could suspect that in the aircraft case, where spatial resolution is not an issue, the benefit of the high frequency channel is marginal. For a spaceborne system, the situation is different, however. One could conceive a retrieval where the highest frequency channel (i.e., best ground resolution) is the prime channel weather permitting, and the lower frequency channels take over in nonfavorable weather conditions, at the same time compromising ground resolution. Even lower than $\mathrm{Ku}$ band would seem reasonable in order to cope with a wider range of weather conditions.

As to the important question: which radiometric sensitivity is required in order not to unduly compromise wind direction retrievals, the present work does not provide a straight answer. As mentioned in Section VI, the retrieval is based on data integrated over $600 \times 600 \mathrm{~m}$ cells, and the corresponding radiometer sensitivity is some $0.05 \mathrm{~K}$. But in Section VII it is argued, based on the circle flight data, that typical measurement errors are around $\pm 0.2 \mathrm{~K}$ ("geophysical noise": the resolution cell is still not very much larger than structures on the wind driven sea surface). Based on a rule of thumb, the $\pm 0.2 \mathrm{~K}$ noise corresponds to a standard deviation of some $0.14 \mathrm{~K}$, i.e., three times the radio- metric noise. Thus, the present retrieval is not radiometric noise limited, and it is felt that a factor of two worse radiometric noise, i.e., some $0.1 \mathrm{~K}$, would serve well, that is, not degrade the retrieval accuracies presented here.

\section{REFERENCES}

[1] S. H. Yueh, W. J. Wilson, S. V. Nghiem, F. K. Li, and W. B. Ricketts, "Polarimetric measurements of sea surface brightness temperatures using an aircraft K-band radiometer," IEEE Trans. Geosci. Remote Sensing, vol. 33, pp. 85-92, Jan. 1995.

[2] S. H. Yueh, W. J. Wilson, S. V. Nghiem, F. K. Li, and W. B. Ricketts, "Polarimetric brightness temperatures of sea surfaces measured with aircraft K- and Ka-band radiometers," IEEE Trans. Geosci. Remote Sensing, vol. 35, pp. 1177-1187, Sept. 1997.

[3] N. Skou and B. Lauersen, "Measurement of ocean wind vector by an airborne, imaging polarimetric radiometer," Radio Sci., vol. 33, pp. 669-675, May-June 1998.

[4] S. H. Yueh, W. J. Wilson, S. J. Dinardo, and F. K. Li, "Polarimetric microwave brightness signatures of ocean wind directions," IEEE Trans. Geosci. Remote Sensing, vol. 37, pp. 949-959, Mar. 1999.

[5] J. R. Piepmeier, A. J. Gasiewski, M. Klein, V. Boehm, and R. C. Lum, "Ocean surface wind direction measurement by scanning polarimetric microwave radiometry," in Proc. Int. Geoscience and Remote Sensing Symp. '98, Seattle, WA, p. 2307.

[6] N. Skou, B. Laursen, and S. Søbjerg, "Polarimetric radiometer configurations: Potential accuracy and sensitivity," IEEE Trans. Geosci. Remote Sensing, vol. 37, pp. 2165-2171, Sept. 1999.

[7] A. J. Gasiewski and D. B. Kunkee, "Calibration and application of polarization-correlating radiometers," IEEE Trans. Microwave Theory Tech., vol. 41, pp. 767-773, May 1993.

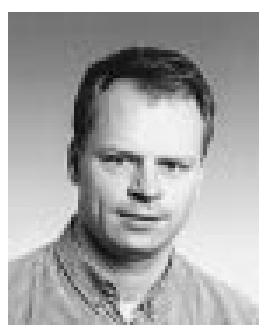

Brian Laursen (M'97) received the M.Sc. and Ph.D. degrees from the Technical University of Denmark, Lyngby, in 1992 and 1999, respectively.

He is currently with Nokia, Denmark. His research has focused on microwave radiometer systems. Correlation radiometry has been his main interest, using the correlation technique in two-dimensional (2-D) synthetic aperture radiometry and in polarimetric radiometry. Ocean surface signatures have been investigated by imaging airborne polarimetric radiometers with the aim of determining wind directions.

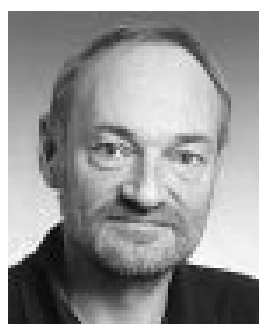

Niels Skou (S'78-M'79-SM'96) received the M.Sc., Ph.D., and D.Sc. degrees from the Technical University of Denmark, Lyngby, in 1972, 1981, and 1990, respectively.

$\mathrm{He}$ is currently an Associate Professor with the Technical University of Denmark. His research has been directed toward microwave remote sensing systems. After working for three years with the development of radar systems for measuring the ice sheets in Greenland and Antarctica, his interest turned toward microwave radiometry. From 1975 to 1979, he developed a scanning, multifrequency, airborne radiometer system. After that, his subjects were radiometer measurements of sea ice and oil pollution on the sea, spaceborne radiometer systems, and development of new systems for specific purposes. In the 1980s, his interest turned back to active instruments, and he became engaged in the development of an airborne, multifrequency, polarimetric and interferometric syntetic aperture radar system. However, activity within microwave radiometry has continued, mainly within the areas of synthetic aperture radiometry and polarimetric radiometry. The work on synthetic aperture radiometry has led to the proposal concerning a soil moisture and ocean salinity (SMOS) mission accepted as one of ESA's Earth Explorer Opportunity Missions. The work with polarimetic radiometry has led to a successful demonstration of a potential new instrument for measuring ocean winds from space. 